\section{Delayed massive hemothorax requiring surgery after blunt thoracic trauma over a 5-year period: complicating rib fracture with sharp edge associated with diaphragm injury}

\author{
Sung Wook Chang, Kyoung Min Ryu, Jae-Wook Ryu \\ Trauma Center, Department of Thoracic and Cardiovascular Surgery, Dankook University Hospital, Cheonan, \\ Korea
}

Delayed massive hemothorax requiring surgery is relatively uncommon and can potentially be life-threatening. Here, we aimed to describe the nature and cause of delayed massive hemothorax requiring immediate surgery. Over 5 years, 1,278 consecutive patients were admitted after blunt trauma. Delayed hemothorax is defined as presenting with a follow-up chest radiograph and computed tomography showing blunting or effusion. A massive hemothorax is defined as blood drainage $>1,500 \mathrm{~mL}$ after closed thoracostomy and continuous bleeding at $200 \mathrm{~mL} / \mathrm{hr}$ for at least four hours. Five patients were identified all requiring emergency surgery. Delayed massive hemothorax presented $63.6 \pm 21.3$ hours after blunt chest trauma. All patients had superficial diaphragmatic lacerations caused by the sharp edge of a broken rib. The mean preoperative chest tube drainage was $3,126 \pm 463 \mathrm{~mL}$. We emphasize the high-risk of massive hemothorax in patients who have a broken rib with sharp edges.

Keywords Thoracic injuries; Hemothorax; Diaphragm; Rib fractures

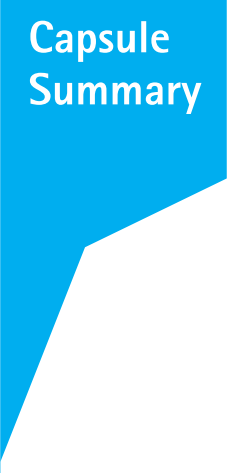

What is already known

Thoracic trauma is a leading cause of mortality, and massive hemothorax is one of the major causes. Generally, massive hemothorax is commonly observed at admission or immediately after trauma. In most cases, massive intrathoracic bleeding is the main indicator for immediate surgery, whereas a retained hemothorax or minor diaphragmatic injuries are indicators for delayed surgery. Therefore, delayed massive hemothorax requiring emergency surgery is relatively uncommon, although it can be potentially life-threatening.

What is new in the current study

The purpose of this study is to describe the nature of delayed massive hemothorax requiring immediate surgery and to identify the major cause.
elSSN: 2383-4625

Received: 26 September 2017

Revised: 28 January 2018

Accepted: 20 February 2018

Correspondence to: Jae-Wook Ryu Department of Thoracic and Cardiovascular Surgery, Dankook University Hospital, 201 Manghyang-ro, Dongnam-gu, Cheonan 31116, Korea E-mail:j3thorax@chol.com

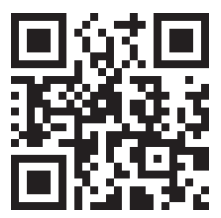

How to cite this article:

Chang SW, Ryu KM, Ryu JW. Delayed massive hemothorax requiring surgery after blunt thoracic trauma over a 5 -year period: complicating rib fracture with sharp edge associated with diaphragm injury. Clin Exp Emerg Med 2018;5(1):60-65.

This is an Open Access article distributed under the terms of the Creative Commons Attribution Non-Commercial License (http:// creativecommons.org/licenses/by-nc/4.0/). 


\section{INTRODUCTION}

Thoracic trauma is a leading cause of morbidity and mortality, and massive hemothorax is one of the major causes. 'Generally, massive hemothorax is caused by injury to the intercostal artery, laceration of the lung, great vessel injury, or diaphragmatic rup-
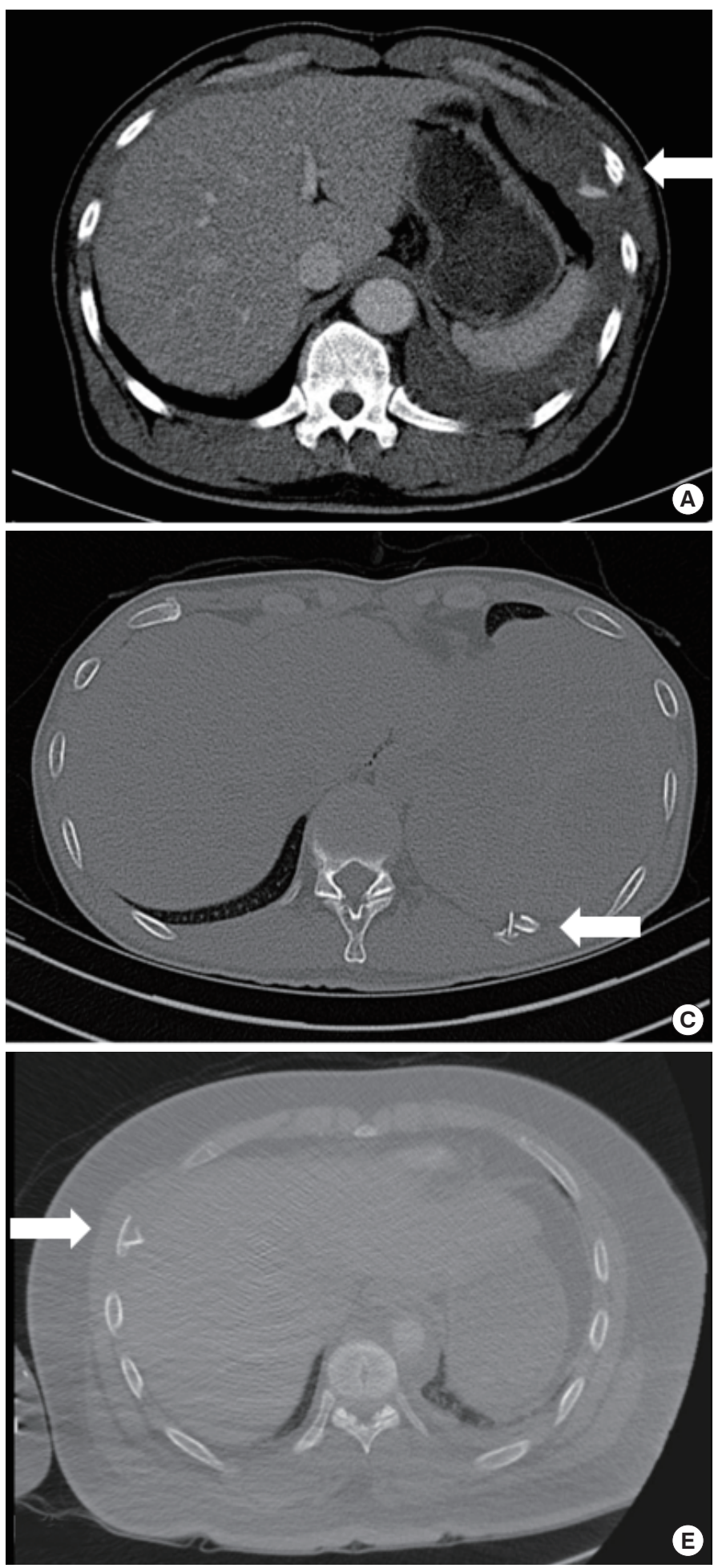

ture, and is commonly observed at admission or immediately after trauma. ${ }^{1,2}$ Delayed hemothorax can be caused by a diaphragm injury or bone bleeding from a fractured rib and is observed after a certain period. ${ }^{3}$ Treatment decisions, such as surgery versus conservative management, depend on the correct evaluation of the patient symptoms. ${ }^{1,3}$ In most cases, massive intrathoracic bleed-
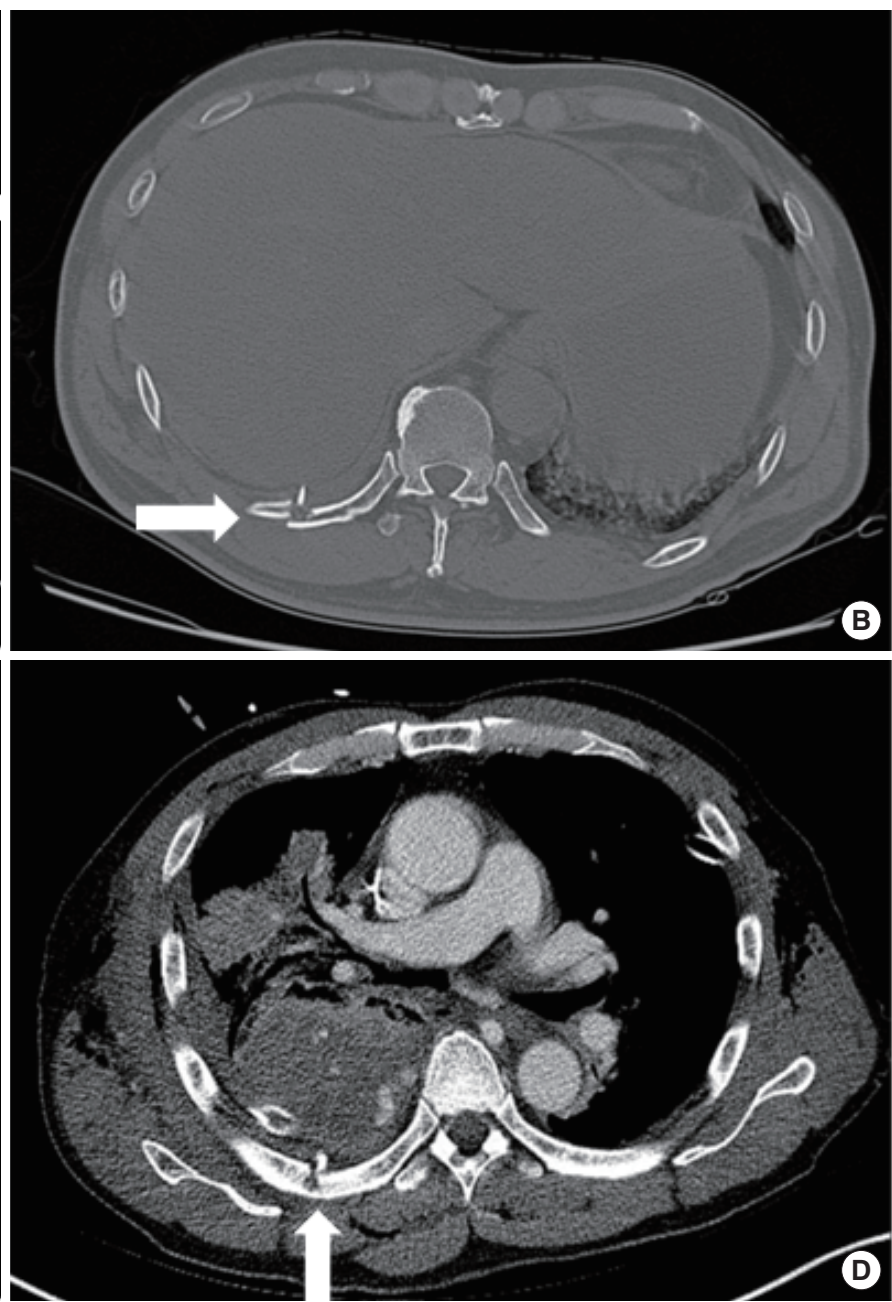

Fig. 1. Chest computed tomography (CT) after blunt thoracic trauma showing a fractured rib with a sharp edge (arrows). (A) Patient 1. Delayed hemothorax and extravasation seen 93 hours after the initial CT. (B) Patient 2. CT showing only a fractured rib with no hemothorax 7 hours later. (C) Patient 3. Delayed hemothorax seen 66 hours later. (D) Patient 4. Delayed hemothorax and extravasation seen 63 hours after the initial CT. (E) Patient 5. CT showing left hemothorax and periaortic hematoma 2 hours later. 
ing is the main indicator for immediate surgery, whereas a retained hemothorax or minor diaphragmatic injuries are indicators for delayed surgery. Therefore, delayed massive hemothorax requiring emergency surgery is relatively uncommon, although it can potentially be life-threatening. ${ }^{2,4-6}$ The purpose of this study is to describe the nature of delayed massive hemothorax requiring immediate surgery and to identify its major cause.

\section{CASE REPORTS}

From February 2011 to January 2015, 1,278 patients were admitted to our hospital after blunt thoracic trauma, 25 of which required operation due to hemothorax. Delayed hemothorax is defined as presenting with a follow-up chest radiograph and computed tomography (CT) showing blunting or effusion for which a normal upright chest radiograph performed at admission or immediately after the trauma did not show costophrenic angle blunting and no pleural effusion had been detected by a chest CT. Massive hemothorax is defined as blood drainage $>1,500 \mathrm{~mL}$ after closed thoracostomy and continuous bleeding at the rate of 200 $\mathrm{mL} / \mathrm{hr}$ for at least four hours. The data were presented as mean \pm standard deviation. Twenty patients who were operated on due to acute massive hemothorax occurring immediately after the trauma or had retained hemothorax were excluded from the study. Their associated injuries were recorded according to the abbreviated injury score (AIS) and injury severity score (ISS) following the 2008 update. The ISS was manually calculated using the AIS handbook.

A total of five patients with delayed massive hemothorax were admitted to our hospital. All patients were men and had chest pain with dyspnea. Three patients were transferred from a different hospital and two presented at our emergency department after trauma. Each patient had a different mechanism of injury, namely, falling, slipping, motorcycle accident, pedestrian traffic accident, and driver traffic accident. Three patients had concurrent extrathoracic injuries: one tibiofibular fracture; one clavicle fracture with liver and scalp lacerations; and one aortic dissection, pelvic bone fracture, liver lacerations, and a deep laceration on the knee. A chest CT was performed for each patient (Fig. 1). The ISS was $22 \pm 7.1$ (range, 16 to 33 ).

Three patients had right hemothorax and two patients had left hemothorax. Four patients underwent closed thoracostomy at our hospital. Among them, three were hemodynamically unstable af-

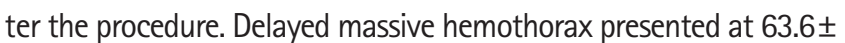
21.3 hours (range, 33 to 93 hours) after blunt thoracic trauma. The mean preoperative chest tube drainage was $3,126 \pm 463 \mathrm{~mL}$ (range, 2,700 to 3,730 $\mathrm{mL}$ ). Before surgery, all patients had an acute-

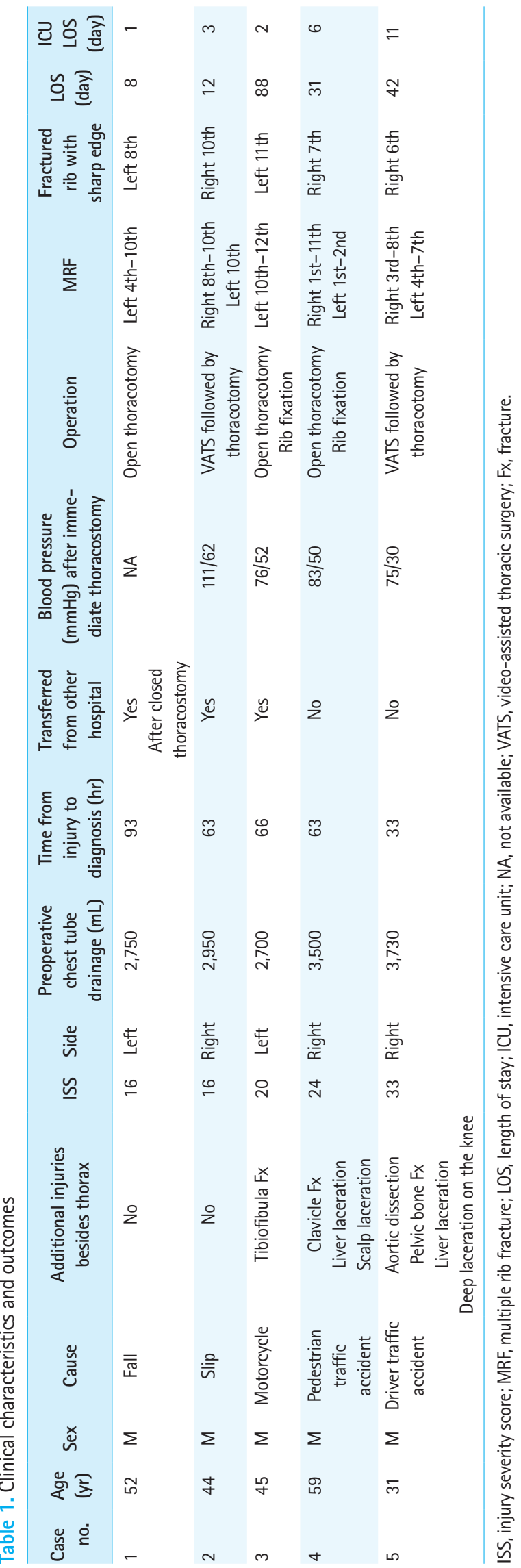



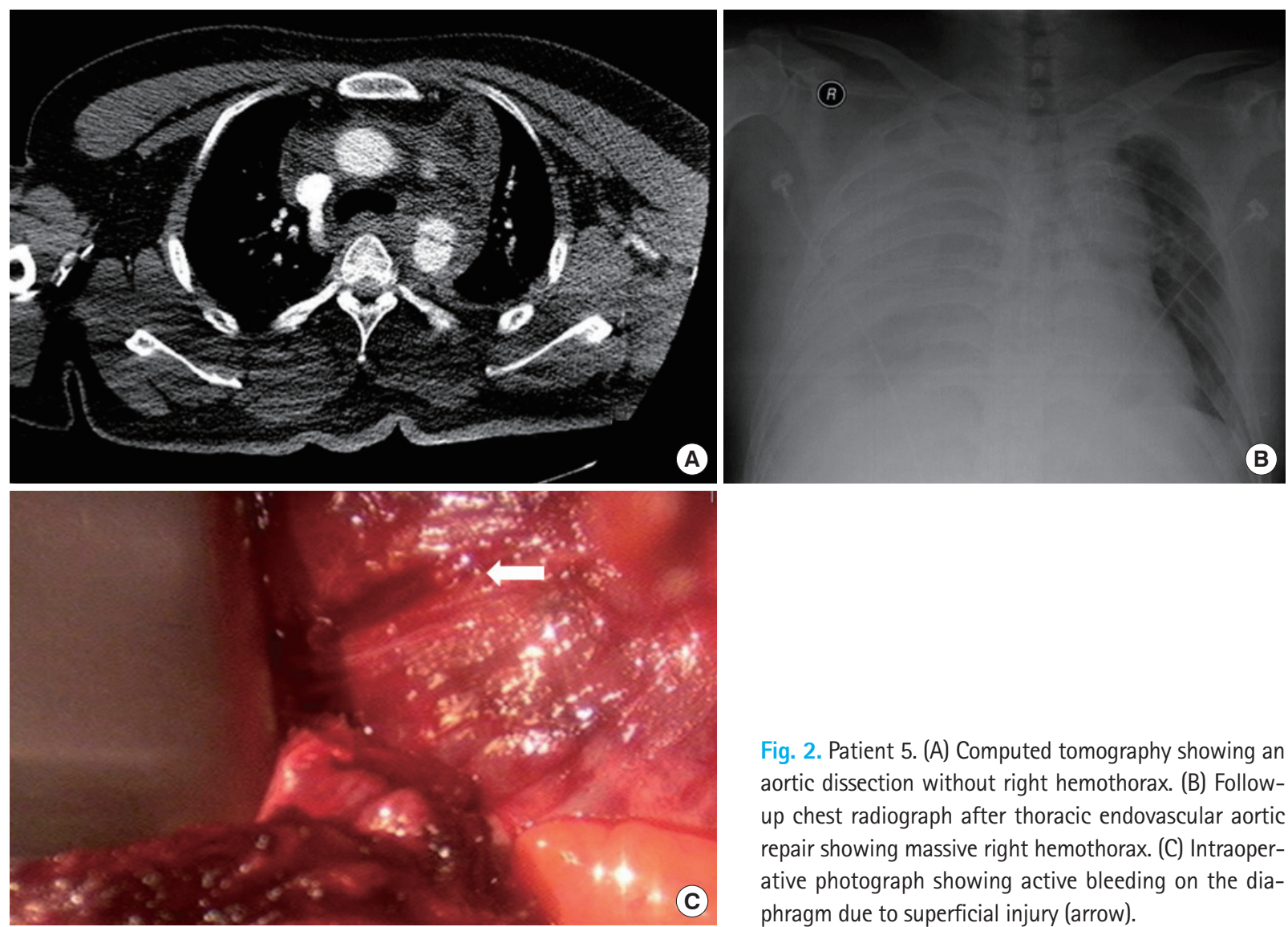

Fig. 2. Patient 5. (A) Computed tomography showing an aortic dissection without right hemothorax. (B) Followup chest radiograph after thoracic endovascular aortic repair showing massive right hemothorax. (C) Intraoperative photograph showing active bleeding on the diaphragm due to superficial injury (arrow).

onset, aggravated chest pain with cold sweats after a coughing spell. The patients' clinical characteristics are shown in Table 1.

We performed emergency open thoracotomy in three patients and video-assisted thoracic surgery (VATS) followed by a thoracotomy in two patients. One patient underwent thoracic endovascular aortic repair due to traumatic aortic dissection using heparin (intravenous 3,000 IU bolus). In this case, the patient was confirmed as having delayed massive hemothorax on the right side 16 hours after thoracic endovascular aortic repair (Fig. 2).

All patients had superficial lacerations on the diaphragm without rupture. Diaphragmatic lacerations with ongoing bleeding could be clearly observed after evacuation of hematomas, after which they were repaired. No other massive bleeding focus was observed in any patient. A fractured rib with a sharp edge was observed around a lacerated diaphragm and was consequently resected. In two patients, unstable fractured ribs were fixed using a straight plate (Biomet Microfixation, Jacksonville, FL, USA).

The mean hospital length of stay was $36 \pm 32$ days (range, 8 to 88 days), and the mean length of stay in the intensive care unit was $5 \pm 4$ days (range, 1 to 11 days). All patients had an uneventful recovery after the hemorrhage and were discharged without any complications. The clinical outcomes are shown in Table 1.

\section{DISCUSSION}

Hemothorax can be categorized into two conditions by a time sequence. At the time of admission or immediately after trauma, patients with costophrenic angle blunting observed on an upright chest radiograph or effusion observed on CT are classified as cases of acute hemothorax. Conversely, cases without blunting or effusion on the initial radiograph or $\mathrm{CT}$ are classified as cases of delayed hemothorax. According to the literature, the prevalence of delayed hemothorax accounts for $4.2 \%$ to $12.2 \%$ of all traumatic hemothoraces, and delayed massive hemothorax requiring surgery is rare. ${ }^{2,4,6,8,9}$ In this study, $5(0.4 \%)$ of the 1,278 patients who had rib fractures received surgery due to delayed life-threatening hemothorax after blunt thoracic trauma.

Several authors have reported that delayed massive hemothorax results from intercostal or phrenic artery tearing, laceration of the diaphragm, or fractured ribs. ${ }^{2,45}$ The time delay between the trauma and the pleural effusion varied from 18 hours to 11 days. ${ }^{6,9}$ In our study, delayed massive hemothoraces resulted from superficial lacerations of the diaphragm in all patients with no active bleeding from intercostal or other vascular injuries. Furthermore, the time delay was relatively short at 33 to 93 hours after blunt 
chest trauma because the patients with retained hemothoraces were not enrolled in the study.

The management of delayed hemothorax primarily requires controlling the bleeding and evacuating the hematoma from the thoracic cavity. A tube thoracostomy should be considered to drain blood from the pleural space, and thoracoscopic surgery or an open thoracotomy should be considered for patients with persistent intrathoracic bleeding. Regarding surgical techniques, an emergency thoracotomy is accepted as an adequate maneuver and allows good exposure of the hemothorax in hemodynamically unstable patients; in addition, VATS may be technically feasible and acceptable in stable patients. ${ }^{2,10,11}$ In this study, three patients received emergency open thoracotomy and two patients received VATS and open conversion. The reasons for conversion to a thoracotomy were inability to control hemostasis and aggravation of the hemodynamic derangement during VATS in the operating room.

In all patients, prompt surgery and awareness of the possibility and early recognition of massive hemothorax were more important factors in improving patient survival than the choice of surgical technique. According to another report, almost all patients had a prodrome of new-onset pleuritic chest pain and/or chest discomfort with dyspnea. ${ }^{9}$ In this study, before surgery, all patients experienced an acute onset of ongoing pleuritic chest pain with cold sweats after a coughing spell. Furthermore, the fractured rib with a sharp edge was intraoperatively observed to be near the diaphragm injury. Therefore, the sharp edge of the broken rib appeared to be the cause of the diaphragmatic laceration, and the massive hemothorax was directly associated with excessive diaphragm movements related to a coughing spell with no other arterial bleeding. Thus, if a patient has a prodrome of some indicative conditions, such as pleuritic chest pain, dyspnea, and cold sweats, the physician should not dismiss the symptoms as those of a general chest pain occurring after blunt thoracic trauma, and the possibility of delayed hemothorax should be considered.

This is case report, so there a few considerations. Not all the patients with a fractured rib with a sharp edge were enrolled. Meanwhile, other patients with a fractured rib with a sharp edge who had been excluded from this study recovered uneventfully without the need for surgery, and their condition did not progress to hemothorax. Therefore, the direct cause and the risk factors of delayed massive hemothorax from a fractured rib remain undetermined. Moreover, not all the patients whose chest pain worsened with or without pleuritic pain after trauma were analyzed and reviewed. Therefore, it is not clear whether newly aggravating pleuritic chest pain is a prodrome of delayed massive hemothorax. However, all patients associated with delayed massive hemothorax in this study had a fractured rib with a sharp edge and prodromal symptoms of pleuritic chest pain. Thus, we believe that our review provides useful and relevant information about delayed massive hemothorax.

We can consider the high-risk or probability of massive hemothorax in patients who have a broken rib with sharp edges. Even if the fractured rib is not severely displaced, a sharp edge may injure the diaphragm surface. Therefore, all patients should be informed of the need for a closer observation upon admission if traumatic hemothorax is found on the initial chest radiograph or $\mathrm{CT}$, even if the fractured rib is not severely displaced. In addition, if a patient has a prodrome of pleuritic chest pain, dyspnea, and cold sweats, delayed hemothorax should be considered.

\section{CONFLICT OF INTEREST}

No potential conflict of interest relevant to this article was reported.

\section{REFERENCES}

1. Huber $S$, Biberthaler P, Delhey $P$, et al. Predictors of poor outcomes after significant chest trauma in multiply injured patients: a retrospective analysis from the German Trauma Registry (Trauma Register DGU). Scand J Trauma Resusc Emerg Med 2014;22:52.

2. Chen $\mathrm{CL}$, Cheng YL. Delayed massive hemothorax complicating simple rib fracture associated with diaphragmatic injury. Am J Emerg Med 2014;32:818.

3. Al-Koudmani I, Darwish B, Al-Kateb K, Taifour Y. Chest trauma experience over eleven-year period at al-mouassat university teaching hospital-Damascus: a retrospective review of 888 cases. J Cardiothorac Surg 2012;7:35.

4. Sharma OP, Hagler S, Oswanski MF. Prevalence of delayed hemothorax in blunt thoracic trauma. Am Surg 2005;71:481-6.

5. Misthos P, Kakaris S, Sepsas E, Athanassiadi K, Skottis I. A prospective analysis of occult pneumothorax, delayed pneumothorax and delayed hemothorax after minor blunt thoracic trauma. Eur J Cardiothorac Surg 2004;25:859-64.

6. Yokosuka $T$, Kobayashi T, Fujiogi $M$, et al. Clinical analysis of delayed hemothorax due to diaphragmatic injury treated by emergent video-assisted thoracic surgery. Kyobu Geka 2014; 67:954-8.

7. Association for the Advancement of Automotive Medicine. Abbreviated injury scale 2005: update 2008. Barrington, IL: Association for the Advancement of Automotive Medicine; 2008.

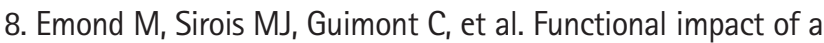


minor thoracic injury: an investigation of age, delayed hemothorax, and rib fracture effects. Ann Surg 2015;262:1115-22.

9. Simon BJ, Chu Q, Emhoff TA, Fiallo VM, Lee KF. Delayed hemothorax after blunt thoracic trauma: an uncommon entity with significant morbidity. J Trauma 1998;45:673-6.
10. Ross RM, Cordoba A. Delayed life-threatening hemothorax associated with rib fractures. J Trauma 1986;26:576-8.

11. Ota H, Kawai H, Matsuo T. Video-assisted minithoracotomy for blunt diaphragmatic rupture presenting as a delayed hemothorax. Ann Thorac Cardiovasc Surg 2014;20 Suppl:911-4. 